\title{
Monolayer diboron dinitride: Direct band-gap semiconductor with high absorption in the visible range
}

\author{
Salih Demirci $\odot,{ }^{1,2}$ Soheil Ershad Rad $\odot,{ }^{1}$ Sahmurat Kazak $\odot,{ }^{1}$ Saffet Nezir, ${ }^{2}$ and Seymur Jahangirov $\odot^{1, *}$ \\ ${ }^{1}$ UNAM-Institute of Materials Science and Nanotechnology, Bilkent University, Ankara 06800, Turkey \\ ${ }^{2}$ Department of Physics, Kırıkale University, Kırlkkale 71450, Turkey
}

(Received 11 November 2019; revised manuscript received 19 February 2020; accepted 24 February 2020; published 9 March 2020)

\begin{abstract}
We predict a two-dimensional monolayer polymorph of boron nitride in an orthorhombic structure (o$\mathrm{B}_{2} \mathrm{~N}_{2}$ ) using first-principles calculations. Structural optimization, phonon dispersion, and molecular dynamics calculations show that o- $\mathrm{B}_{2} \mathrm{~N}_{2}$ is thermally and dynamically stable. o- $\mathrm{B}_{2} \mathrm{~N}_{2}$ is a semiconductor with a direct band gap of $1.70 \mathrm{eV}$ according to calculations based on hybrid functionals. The structure has high optical absorption in the visible range in the armchair direction while low absorption in the zigzag direction. This anisotropy is also present in electronic and mechanical properties. The in-plane stiffness of $\mathrm{o}-\mathrm{B}_{2} \mathrm{~N}_{2}$ is very close to that of hexagonal boron nitride. The diatomic building blocks of this structure hint at its possible synthesis from precursors having B-B and N-N bonds.
\end{abstract}

DOI: 10.1103/PhysRevB.101.125408

Graphene, the two-dimensional (2D) monolayer of carbon, has extraordinary electronic properties [1-4]. Its band structure has two inequivalent Dirac cones at the $K$ and $K^{\prime}$ corners of the Brillouin zone, that makes its charge carriers act as massless Dirac fermions. Defectless ballistic transport makes graphene more conductive than copper [5,6]. Graphene's success has motivated the computational exploration of "beyond graphene" 2D materials [7]. This led to the discovery of group IV monolayers including silicene [8,9], germanene [10], and stanene [11], group III-V [12] and III-VI [13] binary compounds, as well as elemental structures of group III and group V [14]. Borophene, the most studied group III 2D material, has a plethora of allotropes [15], most of which are metallic but there are also semiconducting ones [16]. Pnictogens, 2D allotropes of group $\mathrm{V}$ elements, also come in different geometries such as black [17] and blue [18] phosphorene. It was shown that, if nitrogen atoms are arranged in a buckled hexagonal structure such as blue phosphorene they make a stable structure named nitrogene [19].

Hexagonal boron nitride (h-BN) is another layered material, similar to graphene, which has a highly stable single-layer form, known as white graphene [20]. With a 6-eV band gap [21], 2D h-BN is virtually an insulator. In the bulk form, the boron nitride family consists of four different polymorphic structures, i.e., wurzite (w-BN), cubic (c-BN), rhombohedral $(\mathrm{r}-\mathrm{BN})$, and hexagonal (h-BN). The first and second pairs of these structures have diamondlike $s p^{3}$ and graphitelike $s p^{2}$ hybridized B-N bonds, respectively, while within they differ only in stacking. In addition, a partially disordered phase, turbostratic (t-BN) [22], and a completely disordered phase, amorphous (a-BN) [23], have been reported to be stable. Among these, h-BN is the most abundant form, which

\footnotetext{
*seymur@unam.bilkent.edu.tr
}

was extensively studied to understand the properties of lowdimensional materials. Accordingly, various nanostructures of h-BN were developed, including nanotubes [24], fullerenes [25], nanoribbons [26], etc. Monolayers that deviate from the h-BN structure by making B-B or N-N bonds were also investigated theoretically [27-29].

In this paper, inspired by the aforementioned structures that are formed by the geometric rearrangement of atoms, we propose another stable single-layer structure of boron nitride that is a direct band-gap semiconductor with high absorption in the visible spectrum. In contrast to h-BN, where $\mathrm{B}$ and $\mathrm{N}$ atoms alternate in a hexagonal unit cell, this structure is based on a sequence of $\mathrm{B}-\mathrm{B}$ and $\mathrm{N}-\mathrm{N}$ pairs that are connected to each other in an orthorhombic unit cell. Hence, we named this structure orthorhombic diboron dinitride $\left(\mathrm{o}-\mathrm{B}_{2} \mathrm{~N}_{2}\right)$. Following the description of the first-principles methods used in this study, we report the optimized structural parameters, cohesive energies, a stability analysis based on phonon spectrum calculation and molecular dynamics, the electronic band structure, and the optical and mechanical properties including the in-plane Young's modulus and Poisson's ratio. Finally, we suggest a possible route to synthesis of this structure based on precursors that include $\mathrm{B}-\mathrm{B}$ and $\mathrm{N}-\mathrm{N}$ bonds.

We performed first-principles calculations based on the density functional theory (DFT) using a plane-wave basis set with an energy cutoff of $520 \mathrm{eV}$. We utilize projector augmented-wave potentials [30] and the exchange-correlation potential is approximated by the generalized gradient approximation (GGA) with the Perdew, Burke, and Ernzerhof (PBE) functional [31]. The Brillouin zone (BZ) is represented by an $8 \times 16 \times 1 k$-point grid in the Monkhorst-Pack scheme [32]. The equilibrium configuration of atoms is determined by minimizing the total energy of the system using the conjugate gradient method. The energy convergence criteria between the two consecutive electronic and ionic steps are taken to 
(a)

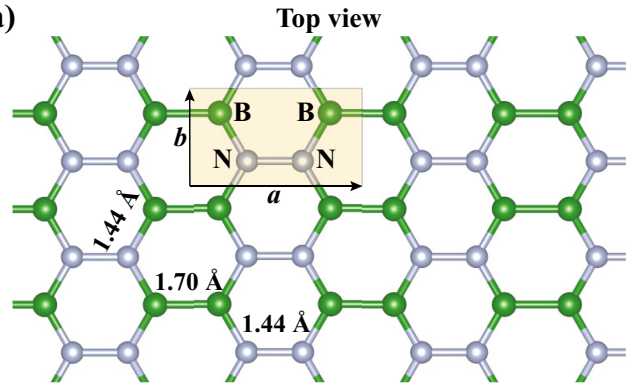

(b)

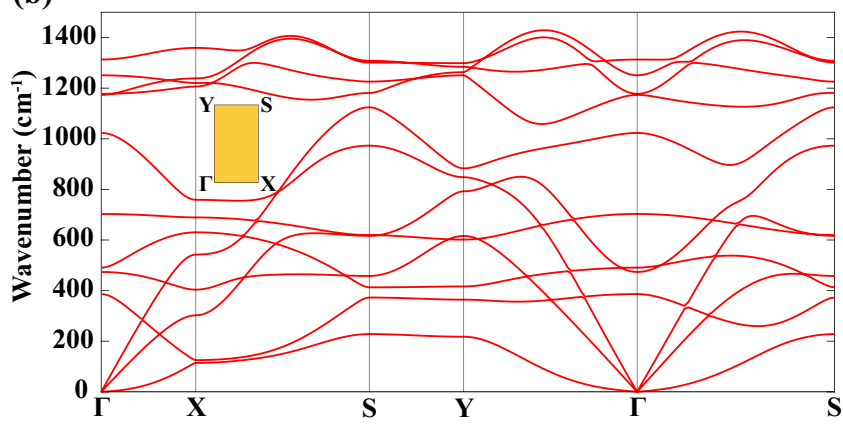

FIG. 1. (a) Top and side view of the ball-and-stick representation of $\mathrm{o}-\mathrm{B}_{2} \mathrm{~N}_{2}$. The orthorhombic unit cell is shaded. (b) The phonon dispersion of o- $\mathrm{B}_{2} \mathrm{~N}_{2}$. The upper right quarter of the Brillouin zone is shown including the visited symmetry points.

be $10^{-5}$ and $10^{-4} \mathrm{eV}$, respectively. In addition, maximum pressure on the lattice has been lowered down to $0.1 \mathrm{kbar}$. To avoid interaction between periodic images in adjacent cells, a vacuum spacing of at least $20 \AA$ was used. All calculations are performed using the Vienna ab initio simulation package (VASP) [33]. Electronic and optical properties are corrected using Heyd-Scuseria-Ernzerhof (HSE06) hybrid functionals [34]. We calculate the phonon spectrum using the density functional perturbation theory (DFPT) [35] with a $3 \times 5 \times 1$ supercell.

The atomic structure of o- $\mathrm{B}_{2} \mathrm{~N}_{2}$ is shown in Fig. 1(a). Upon optimization the Bravais vectors of the orthorhombic unit cell were found to be $a=4.57 \AA$ and $b=2.50 \AA$. The structure consists of B-B and N-N pairs that are connected to each other and lie on the same plane. The lengths of the B-N and N-N bonds are both close to $1.44 \AA$, which is also the value of the $\mathrm{B}-\mathrm{N}$ bond length in h-BN. The B-B bond, on the other hand, is significantly longer, being $1.70 \AA$ A. Hence, the structure can be viewed as stripes of regular hexagons attached to each other while forming elongated hexagons in between. This structural anisotropy also affects the electronic and optical properties, as described later. The calculated value of the cohesive energy of $\mathrm{o}-\mathrm{B}_{2} \mathrm{~N}_{2}$ is $12.54 \mathrm{eV}$ per B-N pair, which is $1.64 \mathrm{eV}$ less than that of h-BN.

The stability of $\mathrm{o}-\mathrm{B}_{2} \mathrm{~N}_{2}$ is first investigated by the calculation of phonon dispersions presented in Fig. 1(b). The square of vibrational frequencies is positive throughout the Brillouin zone, which indicates that there is a restoring force for any small perturbation that displaces atoms from their equilibrium positions. The $D_{2 h}$ symmetry of the o- $\mathrm{B}_{2} \mathrm{~N}_{2}$ structure implies that all the modes are nondegenerate at the gamma point. By analyzing the symmetry of the eigenvectors of each
TABLE I. The optical mode frequencies of the $\mathrm{o}-\mathrm{B}_{2} \mathrm{~N}_{2}$ structure vs their irreducible representation which determine their Raman/IR activity based on the symmetry.

\begin{tabular}{lcc}
\hline \hline & Frequency $\left(\mathrm{cm}^{-1}\right)$ & Symmetry \\
\hline Raman Active & 1312 & $A_{g}$ \\
1253 & $B_{1 g}$ \\
1022 & $A_{g}$ \\
702 & $B_{2 g}$ \\
& 473 & $B_{1 g}$ \\
& 385 & $B_{2 g}$ \\
IR Active & 1178 & $B_{2 u}$ \\
& 1173 & $B_{3 u}$ \\
& 490 & $B_{1 u}$ \\
\hline \hline
\end{tabular}

vibrational mode in the spectrum, the corresponding irreducible representation can be assigned to them. In a structure with $D_{2 h}$ symmetry, modes with $A_{g}, B_{1 g}, B_{2 g}$, and $B_{3 g}$ symmetries correspond to quadratic functions, which make them Raman active, while modes with $B_{1 u}, B_{2 u}$, and $B_{3 u}$ symmetries correspond to linear functions and hence are IR active. This analysis reveals that the Raman spectrum of the o- $\mathrm{B}_{2} \mathrm{~N}_{2}$ structure contains six peaks while the IR spectrum consists of three peaks. The frequencies and the irreducible representations corresponding to these modes are summarized in Table I. A successful synthesis of the $\mathrm{o}-\mathrm{B}_{2} \mathrm{~N}_{2}$ structure should yield similar Raman and IR spectra. The coexistence of optical and acoustical modes at most of the frequency ranges is a sign of vigorous acoustic-optical scattering, which can result in low thermal conductivity [13].

The stability of the proposed structure was put to a further test via molecular dynamics calculations. A $(3 \times 5 \times 1)$ supercell was chosen to avoid constraints imposed by the small unit cell. The temperature was raised from 100 up to $1000 \mathrm{~K}$ in $6 \mathrm{ps}$, and then the system was kept at a constant temperature of $1000 \mathrm{~K}$ for $10 \mathrm{ps}$. The time step was taken to be $2 \mathrm{fs}$ and every 50 steps the velocities were rescaled to match the desired temperature. Throughout these calculations the structure kept its stability without any significant deformation. We note that although a few picoseconds may not grant the appearance of all possible statistical fluctuations, these molecular dynamics results together with the aforementioned phonon dispersion analysis are promising signs of the stability of the $0-\mathrm{B}_{2} \mathrm{~N}_{2}$ structure. We also checked the tendency for o- $\mathrm{B}_{2} \mathrm{~N}_{2}$ to form covalent bonding when they are put on top of each other. Our calculations show that even when two layers are located as close as $2 \AA$, they tend to deflect each other. This is the case for all possible stackings even when atoms are moved in a vertical direction to create buckling. In this respect, the interlayer interaction can be considered to be of van der Waals nature.

The calculated electronic band structure and corresponding partial density of states (DOS) are depicted in Fig. 2. The structure is a semiconductor with a direct gap of $0.64 \mathrm{eV}$ at the symmetry point $Y$ according to calculations based on the PBE functional (dashed green lines). The band gap increases to $1.70 \mathrm{eV}$ when the hybrid HSE06 functional is used (solid red lines) while h-BN is a semiconductor with an 
(a)

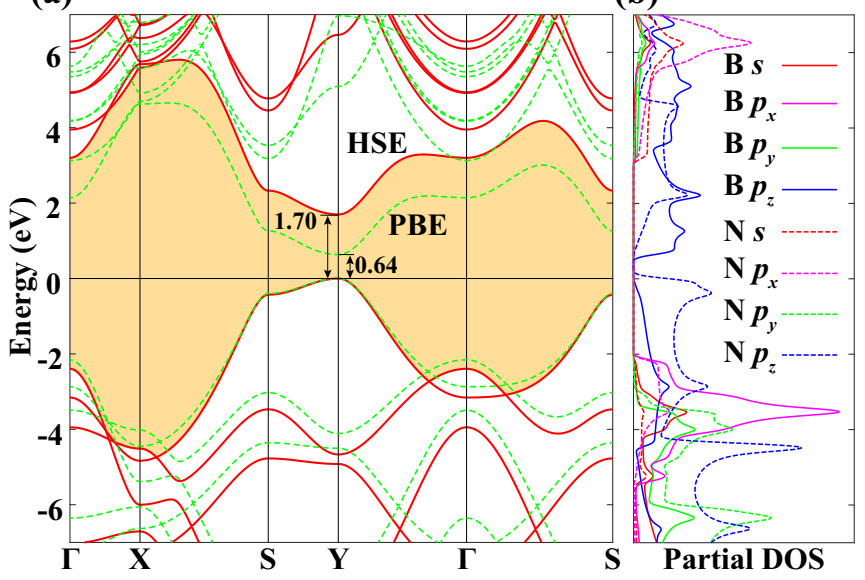

FIG. 2. Electronic band structures of $\mathrm{o}-\mathrm{B}_{2} \mathrm{~N}_{2}$ are calculated by PBE and HSE06 methods, which are shown by dashed green and solid red lines, respectively. (b) Partial density of states (DOS) derived from $\mathrm{PBE}$ calculations projected on $\mathrm{B}$ and $\mathrm{N}$ atoms.

indirect wide band gap of $6 \mathrm{eV}$ [21]. This makes o- $\mathrm{B}_{2} \mathrm{~N}_{2}$ a suitable material for photovoltaic applications such as other direct band-gap 2D materials including $\mathrm{MoS}_{2}$ [36-38], $\mathrm{MoSe}_{2}$ [39], and $\mathrm{WS}_{2}$ [40]. As seen in Fig. 2(b), the valence and conduction band edges are dominated by $\mathrm{N}-p_{z}$ and $\mathrm{B}-p_{z}$ orbitals, respectively. As a consequence of the anisotropy in the structure, the calculated effective masses obtained by fitting of the conduction band minimum are 0.63 and 1.74 in the $Y-S$ and $Y-\Gamma$ directions, respectively. On the other hand, the effective masses of parabolic bands at the $\Gamma$ point around $3-5 \mathrm{eV}$ are around unity in both $k_{x}$ and $k_{y}$ directions. These are nearly-free-electronlike "surface" states [41] that are not affected by the anisotropy of the crystal.

Since both conduction and valence band edges are dominated by $p_{z}$ orbitals, we were motivated to find tight-binding parameters that could be used in further theoretical studies. Here, we follow the formalism used to find the tight-binding parameters of graphene [42]. We consider the same neighborhood of atoms but a variation in the atomic species and bond types results in a model that involves 24 parameters. We find these parameters by fitting the tight-binding bands to three PBE bands that have $p_{z}$ character. Two of these bands form the conduction and the valence band edges while the third one is a valence band around $-6 \mathrm{eV}$. The resulting parameters are presented in Table II. The maximum deviation between the PBE bands used for fitting and the relevant tight-binding bands along the $\Gamma-X-S-Y-\Gamma-S$ path was minimized down to $0.03 \mathrm{eV}$.

The direction-dependent optical absorption properties of $\mathrm{o}-\mathrm{B}_{2} \mathrm{~N}_{2}$ are presented in Fig. 3. Here, the imaginary part of the frequency-dependent dielectric function corresponds to optical absorption which is calculated using the random phase approximation (RPA) with $112 \times 224 \times 1 k$ points and 56 bands. The local field effects are included at the Hartree level only [43]. Results obtained at the PBE level are then corrected using the scissors operation based on the $1.06 \mathrm{eV}$ difference between the PBE and HSE band gaps. This is represented as RPA + Scissors in Fig. 3. We also
TABLE II. Tight-binding parameters for $\mathrm{o}-\mathrm{B}_{2} \mathrm{~N}_{2}$ found by fitting to the PBE band structure. Maximum deviation from the bands used in fitting is $0.03 \mathrm{eV}$.

\begin{tabular}{lccc}
\hline \hline Distance $(\AA)$ & Atoms & Hopping $(\mathrm{eV})$ & Overlap \\
\hline 0 & B, on site & -0.503 & 1 \\
0 & N, on site & -4.375 & 1 \\
1.438 & N-N & 3.382 & 0.406 \\
1.439 & B-N & 2.614 & 0.363 \\
1.701 & B-B & 1.237 & 0.367 \\
2.489 & B-N & 1.056 & 0.044 \\
2.496 & B-B & 1.276 & 0.048 \\
2.496 & N-N & 0.713 & 0.035 \\
2.720 & B-N & 0.621 & 0.014 \\
2.870 & B-B & 0.358 & 0.021 \\
2.880 & N-N & 0.269 & 0.014 \\
3.021 & B-B & 0.190 & 0.012 \\
3.133 & N-N & 0.111 & 0.010 \\
\hline \hline
\end{tabular}

present the RPA calculation made on top of the HSE result (RPA+HSE) performed using a smaller $12 \times 24 \times 1 k$-point grid and 56 bands. The anisotropic structure of o- $\mathrm{B}_{2} \mathrm{~N}_{2}$ is also mirrored in the optical absorption. There is high absorption in the $y$ direction concentrated in the energy dense portion of the solar spectrum which peaks around $465 \mathrm{~nm}$ [44] while the absorption in the $x$ direction is rather weak in the visible range. This directionality is also present in the projected charge densities of the conduction and valence band edge

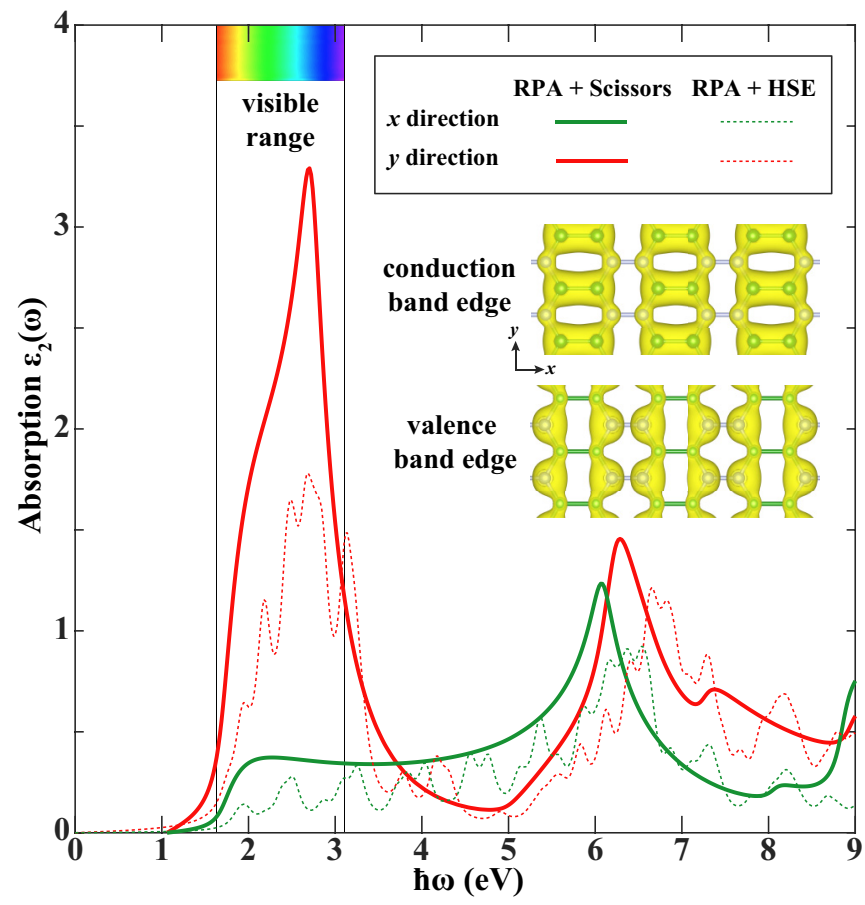

FIG. 3. Direction-dependent optical absorption spectra of o$\mathrm{B}_{2} \mathrm{~N}_{2}$. Solid lines (RPA + Scissors) represent the RPA calculation made on top of PBE and then shifted $1.06 \mathrm{eV}$, which is the difference between the PBE and HSE band gaps. Dotted lines (RPA + HSE) represent the RPA calculation made on top of HSE using a smaller $k$ point grid. Charge densities projected on the conduction and valence band edges are shown as the inset. 
shown as the inset in Fig. 3. Both electronic states are extended along the $y$ axis while localized in $x$. On the other hand, o- $\mathrm{B}_{2} \mathrm{~N}_{2}$ has similar absorption peaks at the ultraviolet range for both the $x$ and $y$ directions [45].

The mechanical properties of $\mathrm{o}-\mathrm{B}_{2} \mathrm{~N}_{2}$ are calculated by applying strain between $-1 \%$ and $+1 \%$ in steps of $0.25 \%$ and the atomic positions are relaxed at each strained step. The elastic strain energy per unit area is expressed using Voigt notation [46] and can be expressed by the following expression,

$$
U\left(\epsilon_{x}, \epsilon_{y}\right)=\frac{1}{2} C_{11} \epsilon_{x}^{2}+\frac{1}{2} C_{22} \epsilon_{y}^{2}+C_{12} \epsilon_{x} \epsilon_{y},
$$

where $\epsilon_{x}\left(\epsilon_{y}\right)$ is strain in the $x(y)$ direction and $C_{11}, C_{22}$, and $C_{12}$ are elastic constants. Due to anisotropy, $C_{11} \neq C_{22}$. For $2 \mathrm{D}$ materials, the mechanical properties are expressed by the in-plane Young's modulus (or stiffness) $(Y)$ and Poisson's ratio $(v)$ which can be written in the $x(y)$ direction as $Y_{x}=\left(C_{11} C_{22}-C_{12} C_{21}\right) / C_{22}\left[Y_{y}=\left(C_{11} C_{22}-C_{12} C_{21}\right) / C_{11}\right]$ and $v_{x}=C_{12} / C_{22}\left(v_{y}=C_{12} / C_{11}\right)$. We first calculated the inplane Young's modulus of graphene (in which case $Y_{x}=Y_{y}$ ) and found it to be $342 \mathrm{~J} / \mathrm{m}^{2}$, which is in good agreement with the value in the literature, $340 \mathrm{~J} / \mathrm{m}^{2}$ [47]. The in-plane Young's modulus $Y_{x}\left(Y_{y}\right)$ and Poisson's ratio $v_{x}\left(v_{y}\right)$ of o- $\mathrm{B}_{2} \mathrm{~N}_{2}$ are found to be 241 (272) J/m $\mathrm{m}^{2}$ and $0.14(0.16)$, respectively. These results are comparable to the in-plane Young's modulus and Poisson's ratio of h-BN, which are found to be $275 \mathrm{~J} / \mathrm{m}^{2}$ and 0.22 .

We propose that $\mathrm{o}-\mathrm{B}_{2} \mathrm{~N}_{2}$ can be synthesized through a controlled reaction between two precursors in which the B$\mathrm{B}$ and $\mathrm{N}-\mathrm{N}$ bonds intrinsically exist. Boron has a tendency towards heteroatomic multiple bonding, while it is reluctant towards multiple bonding with itself, and a structure in the form of $\mathrm{RB}=\mathrm{BR}$ has never been isolated [48]. Therefore, the formation of multiple bonding between two borons is unlikely halfway into the synthesizing process. Boron-boron singular bonding, however, is abundant among boron compounds, and has a relatively high bonding energy of $310 \mathrm{~kJ} / \mathrm{mol}$. On the other hand, the B-N bond has a higher energy of $500 \mathrm{~kJ} / \mathrm{mol}$, but based on the Ostwald-Volmer rule, the B-B bonding which has the largest length will form first, because it leads to a structure with lower density [49]. In addition, it was reported that if B-B bonds become available near nitrogen in a chamber, B-B-N-N species will be abundantly formed by tuning the available energy in the system via a low-power pulsed laser [50-52]. Then, it seems possible to settle these species on a proper substrate to form $\mathrm{o}-\mathrm{B}_{2} \mathrm{~N}_{2}$. Accordingly, it was shown experimentally that a reaction between a structure such as hydrazine, which naturally contains the N-N bonding, and a structure with an inherent B-B bond, such as diboron tetrachloride, can result in a chain or ring of -B-B-N-N-B$\mathrm{B}-\mathrm{N}-\mathrm{N}-$ rather than a configuration with alternate boron and nitrogen atoms [53].

This work was supported by the Scientific and Technological Research Council of Turkey (TÜBİTAK) under Project No. 118F097. S.J. acknowledges support from the Turkish Academy of Sciences - Outstanding Young Scientists Award Program (TÜBA-GEBIP). Part of the computational resources is provided by the National Center for High Performance Computing of Turkey (UHeM) under Grant No. 5003622015. S.D. thanks UNAM, National Nanotechnology Research Center at Bilkent University for the hospitality.
[1] K. S. Novoselov, A. K. Geim, S. V. Morozov, D. Jiang, Y. Zhang, S. V. Dubonos, I. V. Grigorieva, and A. A. Firsov, Science 306, 666 (2004).

[2] K. S. Novoselov, A. K. Geim, S. V. Morozov, D. Jiang, M. I. Katsnelson, I. V. Grigorieva, S. V. Dubonos, and A. A. Firsov, Nature (London) 438, 197 (2005).

[3] Y. Zhan, Y. Tan, H. Stromer, and P. Kim, Nature (London) 438 , 201 (2005).

[4] A. K. Geim and K. S. Novoselov, Nat. Mater. 6, 183 (2007).

[5] P. R. Wallace, Phys. Rev. 71, 622 (1947).

[6] F. Schwierz, Nat. Nanotechnol. 5, 487 (2010).

[7] S. Haastrup, M. Strange, M. Pandey, T. Deilmann, P. S. Schmidt, N. F. Hinsche, M. N. Gjerding, D. Torelli, P. M. Larsen, A. C. Riis-Jensen, J. Gath, K. W. Jacobsen, J. J. Mortensen, T. Olsen, and K. S. Thygesen, 2D Mater. 5, 042002 (2018).

[8] S. Cahangirov, M. Topsakal, E. Aktürk, H. Şahin, and S. Ciraci, Phys. Rev. Lett. 102, 236804 (2009).

[9] P. Vogt, P. De Padova, C. Quaresima, J. Avila, E. Frantzeskakis, M. C. Asensio, A. Resta, B. Ealet, and G. Le Lay, Phys. Rev. Lett. 108, 155501 (2012).

[10] M. E. Dávila, L. Xian, S. Cahangirov, A. Rubio, and G. Le Lay, New J. Phys. 16, 095002 (2014).

[11] F. Zhu, W. Chen, Y. Xu, C. Gao, D. Guan, C. Liu, D. Qian, S. Zhang, and J. Jia, Nat. Mater. 14, 1020 (2015).
[12] H. Şahin, S. Cahangirov, M. Topsakal, E. Bekaroglu, E. Akturk, R. T. Senger, and S. Ciraci, Phys. Rev. B 80, 075305 (2009).

[13] S. Demirci, N. Avazlı, E. Durgun, and S. Cahangirov, Phys. Rev. B 95, 115409 (2017).

[14] F. Ersan, D. Kecik, V. O. Özçelik, Y. Kadioglu, O. Ü. Aktürk, E. Durgun, E. Aktürk, and S. Ciraci, Appl. Phys. Rev. 6, 021308 (2019).

[15] Z. Zhang, E. S. Penev, and B. I. Yakobson, Nat. Chem. 8, 525 (2016).

[16] S. İpek, M. E. Kilic, A. Mogulkoc, S. Cahangirov, and E. Durgun, Phys. Rev. B 98, 241408(R) (2018).

[17] H. Liu, A. T. Neal, Z. Zhu, Z. Luo, X. Xu, D. Tománek, and P. D. Ye, ACS Nano 8, 4033 (2014).

[18] Z. Zhu and D. Tománek, Phys. Rev. Lett. 112, 176802 (2014).

[19] V. O. Özçelik, O. Ü. Aktürk, E. Durgun, and S. Ciraci, Phys. Rev. B 92, 125420 (2015).

[20] L. Liu, Y. P. Feng, and Z. X. Shen, Phys. Rev. B 68, 104102 (2003).

[21] K. Watanabe, T. Taniguchi, and H. Kanda, Nat. Mater. 3, 404 (2004).

[22] S. Alkoy, C. Toy, T. Gönül, and A. Tekin, J. Eur. Ceram. Soc. 17, 1415 (1997).

[23] E. Hamilton, S. Dolan, C. Mann, H. Colijn, C. McDonald, and S. Shore, Science 260, 659 (1993). 
[24] A. Loiseau, F. Willaime, N. Demoncy, G. Hug, and H. Pascard, Phys. Rev. Lett. 76, 4737 (1996).

[25] E. Bengu and L. D. Marks, Phys. Rev. Lett. 86, 2385 (2001).

[26] H. Zeng, C. Zhi, Z. Zhang, X. Wei, X. Wang, W. Guo, Y. Bando, and D. Golberg, Nano Lett. 10, 5049 (2010).

[27] Y. Wang, M. Miao, J. Lv, L. Zhu, K. Yin, H. Liu, and Y. Ma, J. Chem. Phys. 137, 224108 (2012).

[28] J. Li, X. Fan, Y. Wei, and G. Chen, Sci. Rep. 6, 31840 (2016).

[29] K. Waters and R. Pandey, J. Phys.: Condens. Matter 30, 135002 (2018).

[30] P. E. Blöchl, Phys. Rev. B 50, 17953 (1994).

[31] J. P. Perdew, K. Burke, and M. Ernzerhof, Phys. Rev. Lett. 77, 3865 (1996).

[32] H. J. Monkhorst and J. D. Pack, Phys. Rev. B 13, 5188 (1976).

[33] G. Kresse and J. Furthmüller, Phys. Rev. B 54, 11169 (1996).

[34] A. V. Krukau, O. A. Vydrov, A. F. Izmaylov, and G. E. Scuseria, J. Chem. Phys. 125, 224106 (2006).

[35] A. Togo and I. Tanaka, Scr. Mater. 108, 1 (2015).

[36] K. F. Mak, C. Lee, J. Hone, J. Shan, and T. F. Heinz, Phys. Rev. Lett. 105, 136805 (2010).

[37] B. Radisavljevic, A. Radenovic, J. Brivio, V. Giacometti, and A. Kis, Nat. Nanotechnol. 6, 147 (2011).

[38] Y.-H. Lee, X.-Q. Zhang, W. Zhang, M.-T. Chang, C.-T. Lin, K.-D. Chang, Y.-C. Yu, J. T.-W. Wang, C.-S. Chang, J.-L. Li, and T.-W. Lin, Adv. Mater. 24, 2320 (2012).

[39] S. Tongay, J. Zhou, C. Ataca, K. Lo, T. S. Matthews, J. Li, J. C. Grossman, and J. Wu, Nano Lett. 12, 5576 (2012).
[40] H. R. Gutiérrez, N. Perea-López, A. L. Elías, A. Berkdemir, B. Wang, R. Lv, F. López-Urías, V. H. Crespi, H. Terrones, and M. Terrones, Nano Lett. 13, 3447 (2013).

[41] M. Posternak, A. Baldereschi, A. J. Freeman, and E. Wimmer, Phys. Rev. Lett. 52, 863 (1984).

[42] S. Reich, J. Maultzsch, C. Thomsen, and P. Ordejón, Phys. Rev. B 66, 035412 (2002).

[43] H. Ehrenreich and M. H. Cohen, Phys. Rev. 115, 786 (1959).

[44] E. V. Appleton, Nature (London) 156, 534 (1945).

[45] J. Yamaura, Y. Muraoka, T. Yamauchi, T. Muramatsu, and Z. Hiroi, Appl. Phys. Lett. 83, 2097 (2003).

[46] R. C. Andrew, R. E. Mapasha, A. M. Ukpong, and N. Chetty, Phys. Rev. B 85, 125428 (2012).

[47] C. Lee, X. Wei, J. W. Kysar, and J. Hone, Science 321, 385 (2008).

[48] H. Braunschweig and R. D. Dewhurst, Organometallics 33, 6271 (2014).

[49] S. Bohr, R. Haubner, and B. Lux, Diamond Relat. Mater. 4, 714 (1995).

[50] L. Andrews, P. Hassanzadeh, and T. R. Burkholder, J. Chem. Phys. 98, 922 (1993).

[51] P. Hassanzadeh and L. Andrews, J. Phys. Chem. 96, 9089 (1992).

[52] S. Burrill and F. Grein, J. Mol. Struc: THEOCHEM 757, 137 (2005).

[53] A. K. Holliday, F. J. Marsden, and A. G. Massey, J. Chem. Soc. 62, 3348 (1961). 